\title{
Aurora B Inhibitor TAK-901 Synergizes with BCL-xL Inhibition by Inducing Active BAX in Cancer Cells
}

\author{
SAOMI MURAI ${ }^{1}$, JENNIFER MATUSZKIEWICZ $^{2 \ddagger}$, YUUMI OKUZONO $^{1}$, HIROYUKI MIYA $^{1}$ and RON DE JONG ${ }^{2}$ \\ ${ }^{1}$ Pharmacology Research Laboratory II, Pharmaceutical Research Laboratories, \\ Takeda Pharmaceutical Company Ltd., Kanagawa, Japan; \\ ${ }^{2}$ Discovery Biology, Takeda California, Inc., San Diego, CA, U.S.A.
}

\begin{abstract}
Background: Aurora B kinase plays an essential role in chromosome segregation and cytokinesis, and is dysregulated in many cancer types, making it an attractive therapeutic target. TAK-901 is a potent aurora B inhibitor that showed efficacy in both in vitro and in vivo oncology models. Materials and Methods: We conducted a synthetic lethal siRNA screening to identify the genes that, when silenced, can potentiate the cell growth-inhibitory effect of TAK-901. Results: $B$-cell lymphoma-extra large (BCL-xL) depletion by siRNA or chemical inhibition synergized with TAK-901 in cancer cell lines. As a mechanism of synthetic lethality, active BCL2 associated $X$, apoptosis regulator $(B A X)$ was induced by TAK901. BCL-xL protected cells from BAX-dependent apoptosis induction. Therefore, TAK-901 sensitizes cancer cells to BCL$x L$ inhibition. Conclusion: Polyploid cells induced by TAK-901 are vulnerable to $B C L-x L$ inhibition. Our findings may have an impact on combination strategies with aurora $B$ inhibitors in clinical studies.
\end{abstract}

Aurora $\mathrm{B}$ is a component of the chromosomal passenger complex that is essential for chromosome condensation, spindle assembly checkpoint, and cytokinesis $(1,2)$. Aurora $B$ is highly expressed in various human cancer types (3) and

This article is freely accessible online.

${ }^{\ddagger}$ Current address: Quanticel Pharmaceuticals Inc., San Diego, CA, U.S.A.

Correspondence to: Saomi Murai, Pharmaceutical Research Division, Takeda Pharmaceutical Company Limited, 26-1, Muraoka-Higashi 2-chome Fujisawa, Kanagawa 251-8555, Japan. Tel: +81 466321927, e-mail: saomi.murai@takeda.com and Ron de Jong, Takeda California, Inc., Takeda Pharmaceutical Company Limited, 10410 Science Center Dr, San Diego, CA 92121, U.S.A Tel: +1 8587313719, e-mail: ron.dejong@takeda.com

Key Words: Aurora B kinase inhibitor, TAK-901, BCL-xL, synthetic lethal screen. is associated with poor prognosis and progression $(4,5)$, making it an attractive therapeutic target. We previously identified a potent aurora B inhibitor, TAK-901, for the treatment of cancer with aurora B dysregulation (6). It is reported that aurora $\mathrm{B}$ inhibition with a small-molecule inhibitor, such as TAK-901, leads to abnormal chromosome alignment and silencing of the spindle assembly checkpoint. This induces the cells to exit mitosis without completion of cytokinesis, resulting in a polyploid cellular phenotype (6-8). Although aurora B kinase inhibitors induce cell growth inhibition both in vitro and in vivo, some polyploid cells remain after the treatment. Multiple aurora B inhibitors have been tested in clinical trials as a monotherapy with limited success (9); a combination strategy targeting the vulnerability of polyploid cancer cells to aurora B kinase inhibitors may potentiate their therapeutic effects.

The concept of synthetic lethality emerged over a dozen years ago. Targeting of a gene that is synthetically lethal to a cancer-related mutation would specifically suppress cancer cells (10). This strategy enables treatment of cancer with a wide therapeutic index. The concept of synthetic lethality is not only used in mutation-related cancer, but is also applied to explore combination partners, and to identify vulnerability of cancer cells in specific microenvironments in accordance with the increased availability of chemical and genetic tools to interfere with gene function (11-13).

In this study, we conducted a siRNA screen of druggable genes to identify genes indispensable for the survival of polyploid cells, investigated the mechanism of vulnerability of polyploid cells induced by TAK-901, and applied the findings to a combination strategy with TAK-901.

\section{Materials and Methods}

Cell lines and culture. Human cancer cell lines SW480, HCT116, SW620, HT29, U87, A549, H23, H28, H1299, LNCap, H146, H196, H1395, H446, H1688, H209, H510A, and H1048 were purchased from the American Type Culture Collection (Manassas, VA, USA). All cell lines were cultured according to the supplier's recommendations. 
Small molecules and siRNAs. TAK-901 was obtained from Takeda Calfornia, Inc. (San Diego, CA, USA). ABT-263 (14) was purchased from Funakoshi (Tokyo, Japan), and AZD1152-HQPA (15) and gossypol were obtained from Sigma-Aldrich (St. Louis, MO, USA). For the siRNA screening, the Human Druggable Genome siRNA Set Ver. 4.1 was purchased from QIAGEN (Hilden, Germany). SilencerSelect Negative Control No. 2 and BCL2 like 1 (BCL2L1) (s1921, s1922), BAX (s1889, s1890), BCL2 antagonist/killer 1 (BAK) (s1880, s1881), and p53 (s605, s606) SilencerSelect siRNAs were purchased from Thermo Fisher scientific (Waltham, MA, USA).

siRNA transfection. The siRNA screening was conducted by solidphase transfection in a 1536-well plate format (16). Shortly, HCT116 cells were seeded at 150 cells/well in 1536-well plates and dimethyl sulfoxide (DMSO) or $100 \mathrm{nM}$ TAK-901 were added to the wells 24 $\mathrm{h}$ after cell seeding. After $72 \mathrm{~h}$ of incubation, cell proliferation was measured using CellTiter Glo. For individual siRNA experiments, HCT116 cells were seeded at 2,000 cells/well in 96-well plates and simultaneously transfected with siRNAs using Lipofectamine RNAiMAX transfection reagent (Thermo Fisher Scientific, Waltham, MA, USA) according to the manufacturer's recommendations. Individual siRNAs were transfected at a final concentration of $10 \mathrm{nM}$. For simultaneous treatment with siRNA and compounds, cells were transfected with indicated siRNAs for $24 \mathrm{~h}$ followed by a $72-\mathrm{h}$ incubation with each compound at given concentrations.

Cell proliferation and caspase-3/7 assays. For general cell proliferation assay, 5000 cells were seeded in 96-well plates and cultured for $24 \mathrm{~h}$. Then, cells were treated with TAK-901, AZD1152-HQPA or ABT-263 for $72 \mathrm{~h}$. Cell viability was determined using the CellTiter-Glo luminescence assay (Promega, Madison, WI, USA) according to manufacturer's protocol. Luminescent signals were detected using an ARVO MX1420 microplate reader (PerkinElmer, Wellesley, MA, USA). Caspase-3/7 activity was determined using the Caspase-Glo 3/7 Assay (Promega, Fitchburg, WI, USA) per the manufacturer's protocol.

Analysis of combination effect. The Delta Plot Analysis method was used to determine whether the effect of a drug combination treatment was synergistic or additive. The Delta Plot Analysis method uses either a Bliss Independence or Loewe Additivity model. The method is comparative in nature, and results are plotted as a function of cell survival, with confidence intervals for determining significance above/below an additive effect line (17).

Quantitative Reverse Transcription polymerase chain reaction ( $q R T$ $P C R)$ analysis. Following the designated treatment, total RNA was isolated from cells and purified with an RNeasy Mini Kit (Qiagen, Valencia, CA, USA) according to the manufacturer's instructions. qPCR analysis was performed on a ViiA7 (Applied Biosystems, Foster City, CA, USA), using TaqMan Fast Advanced Master Mix with TaqMan probes. Data were analyzed according to the $2-\Delta \Delta \mathrm{Ct}$ method and normalized to the amount of glyceraldehyde-3phosphate dehydrogenase $(G A P D H)$ mRNA. The normalized abundances of target mRNAs were expressed relative to the corresponding values for cells treated with DMSO or negative control siRNAs. The following TaqMan probes were used for quantitative RT-PCR analysis: GAPDH (Hs02786624_g1), BAX (Hs00180269_m1), BCL2L1 (Hs99999146_m1), tumor protein p53
(TP53) (Hs01034249_m1), cyclin-dependent kinase inhibitor 1A (CDKN1A) (Hs00355782_m1), phorbol-12-myristate-13-acetateinduced protein 1 (PMAIP1, NOXA) (Hs00560402_m1), BCL2 binding component 3 (BBC3) (Hs00248075_m1), p53-induced death domain protein 1 (PIDD1) (Hs00611142_g1) (Applied Biosystems, Waltham, MA, USA).

Western blotting. Whole cell extracts were prepared with $1 \times$

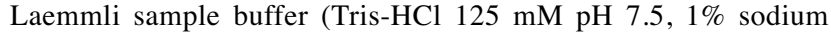
dodecyl sulfate (SDS), $20 \%$ glycerol). Whole cell extracts or immunoprecipitates were fractionated by SDS-poly-acrylamide gel electrophoresis, and transferred to polyvinylidene difluoride membranes using an iBlot Transfer Stack and iBlot Gel Transfer Device (Invitrogen/Thermo Fisher Scientific, Waltham, MA, USA). After incubation with StartingBlock ${ }^{\mathrm{TM}}$ T20 in phosphate-buffered saline (PBS) Blocking Buffer (Pierce Biotechnology, Rockford, IL, USA), the membranes were incubated overnight with primary antibodies, followed by a 1-h incubation with horseradish peroxidase-conjugated secondary antibodies (Cell Signaling Technology, Beverly, MA, USA). For imaging, the membranes were soaked in ImmunoStar Zeta (Wako, Osaka, Japan) and scanned using an ImageQuant LAS-3000 (Fujifilm, Tokyo, Japan). Mitochondrial fractionation was conducted using Qproteome Mitochondria Isolation Kit (Qiagen). The following antibodies were used for immunoprecipitation or western blot analysis: anti-p53 (DO-1, sc-126) and anti-p21 (C-19, sd-397) from Santa Cruz Biotech (Dallas, TX, USA), anti-BAX (\#2772), anti-BAK (\#3814), anti-pHistoneH3 (\#9715), anti-GAPDH (\#2118), anti-BCL2L1 (53H6, \#2764), and anti-BCL2 family apoptosis regulator (MCL1; \#4572) from Cell Signaling Technologies (Danvers, MA, USA), anti-BAX (6A7, ab5714) from Abcam (Cambrige, UK).

Immunoprecipitation. In general, cells were lysed in RIPA buffer on ice. For immunoprecipitation with BAX 6A7 antibodies (Abcam), cells were lysed in 1\% 3-[(3-cholamidopropyl)dimethylammonio]1-propanesulfonate on ice. For immunoprecipitation, cell lysate was incubated with Dynabeads Protein G (Invitrogen) conjugated with BAX, BCL-xL, or MCL1 antibodies overnight at $4^{\circ} \mathrm{C}$ with gentle rocking. Following three washes with lysis buffer, the bound proteins were eluted from the beads with $2 \times$ Laemmli sample buffer. The immunoprecipitated samples were then analysed by western blotting.

Fluorescence-activated cell sorting (FACS) analysis. HCT116 cells were pelleted, washed twice with PBS, and suspended in $150 \mu \mathrm{l}$ of cold PBS. Cold $100 \%$ ethanol $(350 \mu \mathrm{l})$ was added to the cell suspensions, which were then vortexed and incubated at $-20^{\circ} \mathrm{C}$ for 30 minutes. The cells were pelleted, resuspended in PBS containing $32 \mathrm{ng} / \mu \mathrm{l}$ RNase A (Sigma-Aldrich), and incubated at room temperature for 30 minutes. Propidium iodide was added at a concentration of $40 \mathrm{ng} / \mu \mathrm{l}$, and the cells were incubated for 10 minutes at room temperature. Samples were analysed by FACS for cell-cycle distribution.

\section{Results}

Druggable siRNA screen reveals that BCL-xL knock-down synergizes with TAK-901. As reported previously, enlarged nuclei in HCT116 cells appeared after TAK-901 treatment (6), which demonstrated that TAK-901 induced polyploid 
cells. The phenotype is caused by aurora B inhibition, as confirmed by treatment with AZD1152-HQPA, a selective aurora B kinase inhibitor (Figure 1A). Induction of polyploidy was also detected by FACS analysis; $2 \mathrm{~N}$ and $4 \mathrm{~N}$ cells were observed in DMSO-treated HCT116 cells, while mainly $4 \mathrm{~N}$ and $8 \mathrm{~N}$ were observed in TAK-901- or AZD1152HQPA-treated HCT116 cells (Figure 1B). To identify genes that, when knocked down, synergize with TAK-901, we conducted a screen against a library of 27,921 siRNAs targeting 6,870 druggable genes. The screen was conducted using the solid-phase reverse transfection method (18) on the HCT116 cell line in 1536-well plates (Figure 1C). The screen was performed twice, with high reproducibility (correlation factor $=0.833$ ) (Figure 1D). Hit genes were selected based on the criterion that more than two siRNA sequences satisfied a Z-score of -1.2 or less in two experiments (Figure 1E). In total, 13 genes fulfilled this criterion (Figure 1F). We confirmed the screening results using siRNAs with different sequences from another vendor and found that $B C L-x L$ knock-down synergized well with TAK-901 or AZD1152-HQPA in HCT116 cells (Figure 1G and $\mathrm{H}$ ). In agreement with these observations, a synergistic effect was observed when both aurora $\mathrm{B}$ and $B C L-x L$ were knocked down (Figure 1I). These results suggest that genetic knock-down of $B C L-x L$ synergizes with TAK-901 in HCT116 cells.

Chemical BCL-xL inhibition synergizes with TAK-901 in cancer cell lines. To confirm the synergistic effect of TAK901 with siRNA-mediated $B C L-x L$ knock-down, we performed a combination treatment of TAK-901 or AZD1152-HQPA with ABT-263, a small-molecule BCL2/BCL-xL inhibitor. Synergistic cell growth inhibition of HCT116 cells was observed when TAK-901 and ABT-263 were combined (Figure 2A). Caspase-3/7 activity was also enhanced $24 \mathrm{~h}$ after the combination treatment as compared to each treatment alone, indicating that apoptosis was synergistically induced (Figure 2B). Similar results were observed when using AZD1152-HQPA with ABT-263 (Figure 2C and D). We conducted a larger combinatorial study with 18 human cancer cell lines including colorectal cancer, non-small cell lung cancer small cell lung cancer, and prostate cancer and glioblastoma cells using Delta Plot Analysis, a novel graphical approach (17), to calculate additive or synergistic interactions between TAK-901 and ABT-263. We found that TAK-901 or AZD152-HQPA and ABT-263 synergistically inhibited cell growth in multiple cell lines, regardless of the cancer type (Figure 2E and F). The synergistic effect was also observed when gossypol, a different BCL-xL inhibitor, was combined with TAK-901 (Figure 2G). Overall, these results showed that combined inhibition of aurora $\mathrm{B}$ and $\mathrm{BCL}-\mathrm{xL}$ can result in synergistic cancer cell killing.
TAK-901 increases active BAX at mitochondria p53dependently and induces BAX-dependent apoptosis in combination with ABT-263. To elucidate the mechanism of the combination effect of TAK-901 and ABT-263, we examined the regulation of pro- and anti-apoptotic proteins in HCT116 cells. We found that the protein levels of BAX, p53, and p21, but not BAK, were increased by TAK-901 in HCT116 cells, whereas Ser10 phosphorylation of histone H3, a substrate of aurora B kinase, was suppressed (Figure 3A). mRNA expression of $B A X$ was also increased by TAK-901 (Figure 3B). mRNA expression of $\mathrm{p} 53$ downstream proteins $\mathrm{p} 21$ and PIDD1, but not NOXA and BBC3, was enhanced by TAK-901 treatment (Figure 3C). To confirm that $B A X$ gene induction by TAK-901 was p53-dependent, we investigated BAX expression under the condition of p53 depletion by mRNA knock-down. In the absence of p53, expression of BAX, as well as p21, was reduced at the mRNA and protein levels (Figure 3D and E), indicating that TAK-901 induces BAX expression in a p53-dependent manner in HCT116 cells. Cell growth inhibition by combination treatment of TAK-901 and ABT-263 was relieved by p53 depletion (Figure 3F), suggesting that p53 plays a role in the combination effect.

$B A X$ is key to the synergistic effect and is suppressed by $B C L-x L$. To investigate whether BAX induced by TAK-901 was activated, we immunoprecipitated cellular BAX protein with the BAX 6A7 monoclonal antibody, which recognizes the active BAX protein conformation. TAK-901 slightly increased active BAX monomer as well as total BAX protein in HCT116 cells. Immunoprecipitation of BCL-XL revealed that it was bound to BAX, but this binding was completely abrogated by ABT-263 in HCT116 cells. MCL1 expression was also slightly reduced by TAK-901 treatment. In A549 cells, BAX expression was not increased by TAK-901, but an increase in active $\mathrm{BAX}$ protein and reduced binding to BCL-xL and MCL1 were observed, which is consistent with the findings in HCT116 (Figure 4A). Low levels of BAK protein were found in HCT116 cells, and binding to BCL-xL was not detected (Figure 4B). After TAK-901 treatment, BAX was translocated from the cytoplasm to the mitochondrial membrane (Figure 4C). To confirm that BAX expression is critical for the synergy of TAK-901 and ABT263, $B A X$ expression was suppressed by siRNA in combination with inhibitor treatment. $B A X$ knock-down cancelled the cell growth inhibition and apoptosis induced by TAK-901/ABT-263 or AZD1152-HQPA/ABT-263 combination treatment of HCT116 cells (Figure 4D-G). Furthermore, the synergy was not observed in the BAX-null HCT116 cell line (Figure $4 \mathrm{H}$ and J). Similar results were obtained in combination with AZD1152-HQPA and ABT-263 (Figure 4I and $\mathrm{K}$ ). Therefore, BAX is a critical factor in mediating the synergistic effects of combined inhibition of aurora B kinase and BCL-xL. 


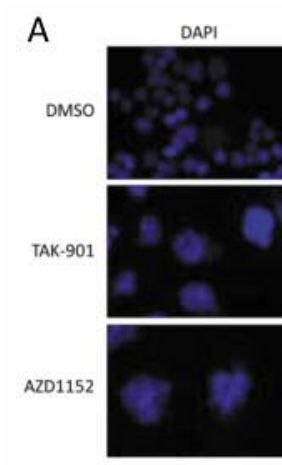

C

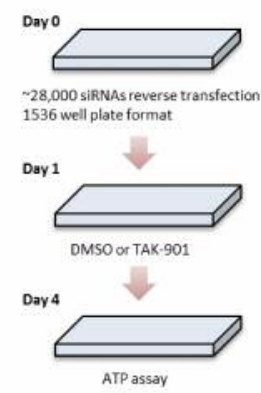

G

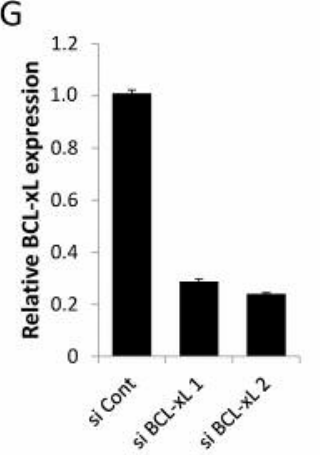

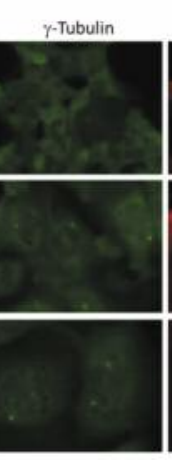

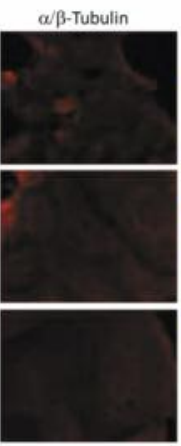

D
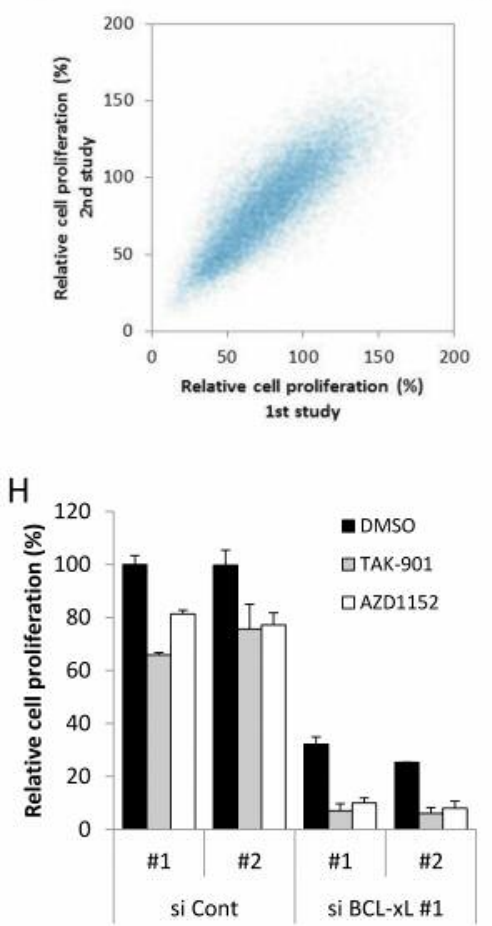

B
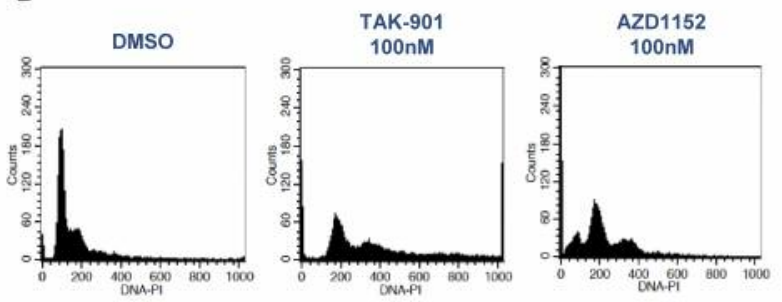

$\mathrm{F}$

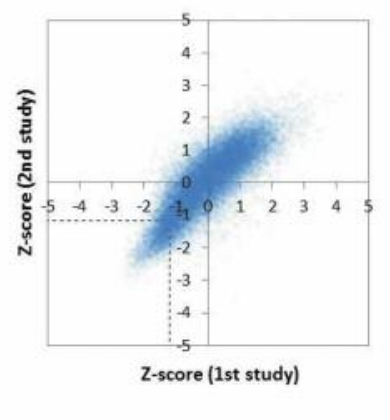

\begin{tabular}{ccc}
\hline \multirow{2}{*}{ Gene symbol } & \multicolumn{2}{c}{ z-score } \\
& 1st & 2nd \\
\hline AKAP14 & -1.601 & -1.221 \\
AKAP14 & -1.465 & -1.484 \\
BCL2L1 & -2.08 & -1.227 \\
BCL2L1 & -2.824 & -2.057 \\
CUBN & -1.201 & -1.746 \\
CUBN & -2.429 & -1.315 \\
EPHA10 & -2.136 & -1.836 \\
EPHA10 & -2.572 & -1.381 \\
LOC220429 & -1.732 & -1.562 \\
LOC220429 & -1.597 & -1.233 \\
MSH5 & -2.333 & -1.472 \\
MSH5 & -2.01 & -1.445 \\
PRKAA2 & -1.248 & -1.429 \\
PRKAA2 & -1.351 & -1.711 \\
PRKACB & -1.902 & -2.156 \\
PRKACB & -1.307 & -1.465 \\
PRL & -1.526 & -1.272 \\
PRL & -1.295 & -1.286 \\
RAF1 & -1.962 & -2.456 \\
RAF1 & -1.966 & -1.409 \\
SLC4A4 & -2.126 & -1.544 \\
SLC4A4 & -1.621 & -1.639 \\
SRPK1 & -1.55 & -2.24 \\
SRPK1 & -1.254 & -1.94 \\
TUBB2A & -1.99 & -1.825 \\
TUBB2A & -1.297 & -2.135 \\
\hline & & \\
& &
\end{tabular}

Figure 1. TAK-901 synthetic lethality screen with druggable gene siRNA library reveals that B-cell lymphoma-extra large (BCL-xL) knock-down synergizes with TAK-901. A: HCT116 cells were treated with 100 nM TAK-901 or 100 nM AZD1152-HQPA for 72 h. After staining with 4',6diamidino-2-phenylindole (DAPI) and anti- $\gamma$-tubuline, anti- $\alpha / \beta$-tubulin, nuclei and tubulins were imaged. B: HCT116 cells were treated with or without 100 nM TAK-901 or $100 \mathrm{nM}$ AZD1152-HQPA for $72 \mathrm{~h}$ and stained with propidium iodide for fluorescence-activated cell sorting analysis. $C$ : Schematic diagram of the siRNA screening process. HCT116 cells were plated in 1536-well plates and reversely transfected with siRNAs. After 24 hours, cells were treated with dimethyl sulfoxide (DMSO) or TAK-901 for $72 \mathrm{~h}$, and cell proliferation was measured. D: Cell growth in DMSOtreated plates from duplicate siRNA screens are plotted. The Spearman correlation coefficient is $r=0.833$. E: Dot plot of Z-scores of siRNA screen from duplicated studies $(r=0.82)$. Dotted line shows $Z$-score $=-1.2$. F: List of hit genes. Criterion for a hit gene was two siRNAs from the same gene with a Z-score of less than -1.2 in duplicated studies. G: HCT116 cells were reversely transfected with BCL- $x L$ siRNA for 48 h and BCL- $x L$ mRNA level was measured by TaqMan analysis. H: HCT116 cells were reversely transfected with BCL-xL siRNA for 24 h, cultured with 100 nM TAK-901 or $100 \mathrm{nM}$ AZD1152-HQPA for $72 \mathrm{~h}$, and cell proliferation was measured. I: HCT116 cells were reversely transfected with BCL-xL siRNA, aurora B siRNA, or their combination. Cell proliferation was measured 96 h after siRNA transfection.

\section{Discussion}

TAK-901 is a potent aurora B inhibitor that causes cell-growth inhibition in multiple cancer cell lines and induces a multinucleated polyploid phenotype. We conducted a synthetic lethal siRNA screen to elucidate the vulnerability of polyploid HCT116 cells treated with TAK-901. Our screen identified BCL-xL as a key molecule rendering cancer cells resistant to 
A

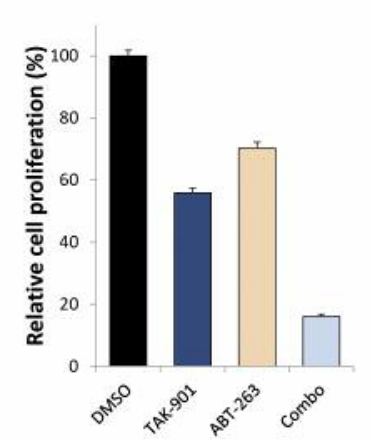

$\mathrm{E}$
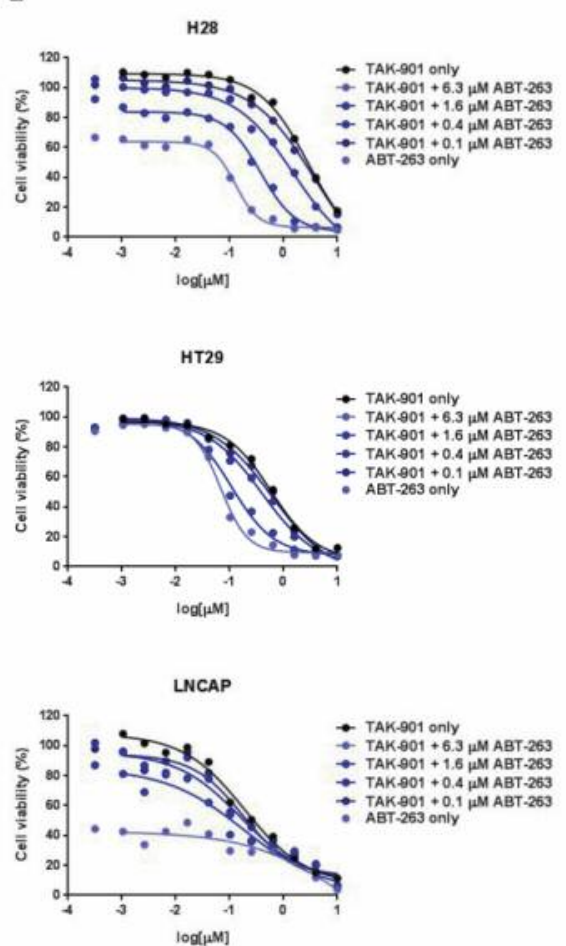

B

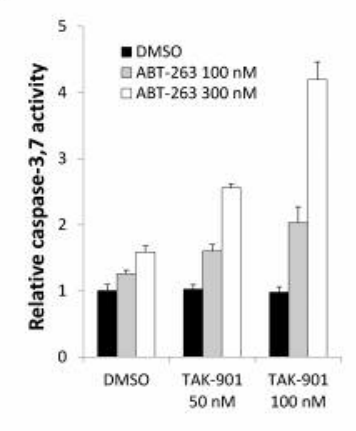

C

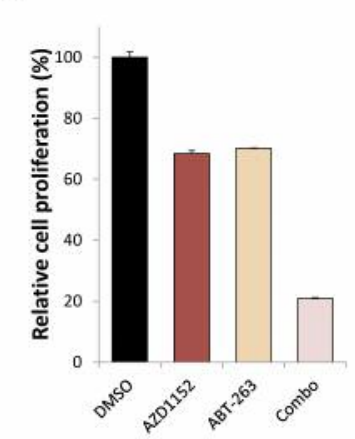

D

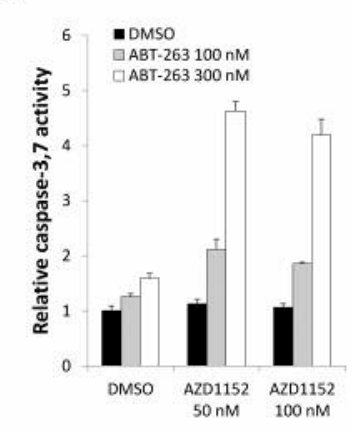

$\mathrm{F}$
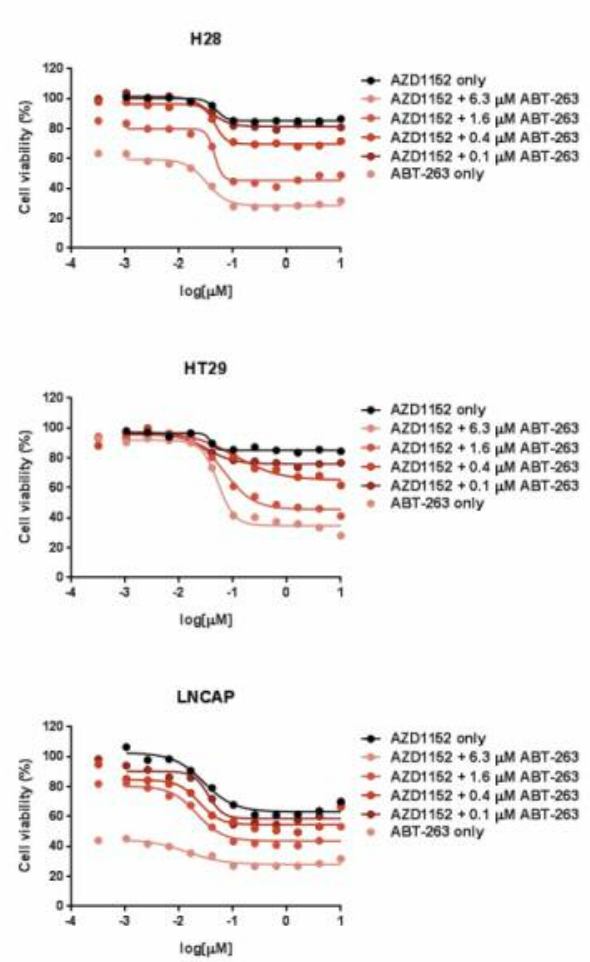

\begin{tabular}{ccc} 
Cell line & Tissue & TAK901+ABT \\
\hline SW480 & CRC & Symergistic \\
HCC 116 & CRC & Symergistic \\
SW620 & CRC & Some synergy \\
HT29 & CRC & Symergistic \\
U-87 MG & Glioblastoma & Some symergy \\
A549 & NSCLC & Symergistic \\
NCI-H23 & NSCLC & Additive \\
NCI-H28 & NSCLC & Synergistic \\
NCI-H1299 & NSCLC & Additive \\
LNCaP & Prostate & Additive \\
NCI-H146 & SCLC & Additive \\
NC1-H196 & SCLC & Additive \\
NCI-H1395 & SCLC & Additive \\
NCI-H446 & SCLC & Additive \\
NCI-H1688 & SCLC & Additive \\
NCI-H209 & SCLC & Synergistic \\
NCL-H510A & SCLC & Symergistic \\
NCI-H1048 & SCLC & Some synergy \\
\hline
\end{tabular}

G

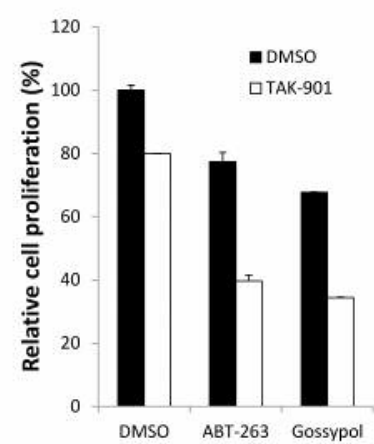

Figure 2. Inhibition of B-cell lymphoma-extra large (BCL-xL) with ABT-263 is sufficient for synergistic cytotoxicity with TAK-901. A, C: HCT116 cells were treated with $100 \mathrm{nM}$ TAK-901 or $100 \mathrm{nM}$ AZD1152-HQPA alone or in combination with $250 \mathrm{nM}$ ABT-263 for $72 \mathrm{~h}$ and cell proliferation was measured. B, D: HCT116 cells were treated with TAK-901 or AZD1152-HQPA alone in combination with ABT-263 at the indicated concentrations for 24 hours and caspase-3,7 activity was measured. E: The effects of the combination of TAK-901 and ABT-263 were examined in several types of cancer cell lines. The combination effect was determined as described in the Material and Methods section. F: Table summarizing the TAK-901 + ABT-263 combination effect on cancer cell lines. G: HCT116 cells were treated with TAK-901 and ABT-263 or gossypol for 72 h, and cell proliferation was measured. CRC: colorectal cancer; SCLC: small cell lung cancer; NSCLC: non-small cell lung cancer.

TAK-901-induced cell killing. TAK-901 suppressed cellular phosphorylation of histone $\mathrm{H} 3$, which is a direct substrate of aurora B kinase, and induced polyploidy with p53 activation in HCT116 cells. p53 is known to be a guardian that inhibits tumourigenesis by eliminating tetraploid or aneuploid cells, which are hallmarks of solid tumours (19). TAK-901 may make cancer cells more prone to undergo apoptosis by inducing polyploidy and subsequent p53 activation.
Our results showed that BAX is induced by TAK-901 in a p53-dependent manner in HCT116 cells. Furthermore, the active form of BAX was increased and translocated to the mitochondrial membrane by TAK-901. BAX is known to be a pro-apoptotic protein and when activated, it localizes to the mitochondrial membrane where it forms oligomers which induce mitochondrial outer-membrane permeabilization and apoptosis (20). Therefore, TAK-901 
A

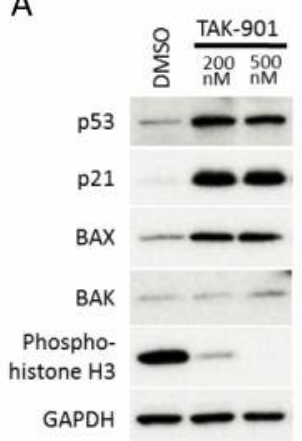

D

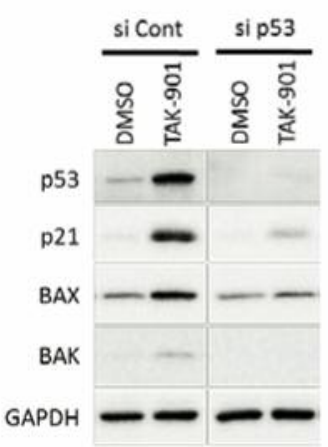

B

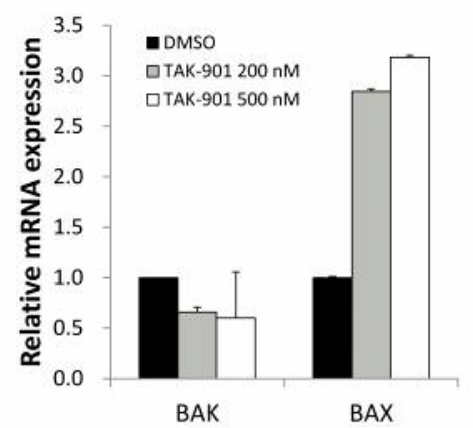

E

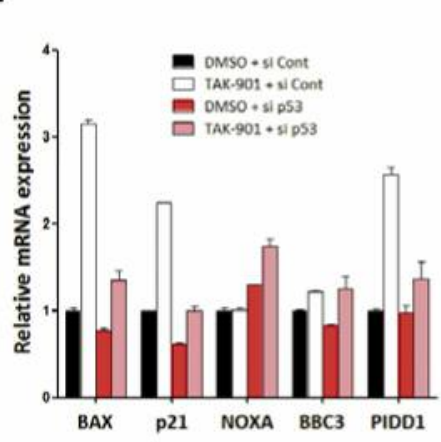

C

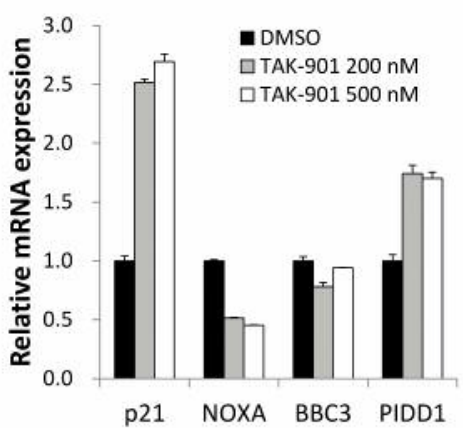

$\mathrm{F}$

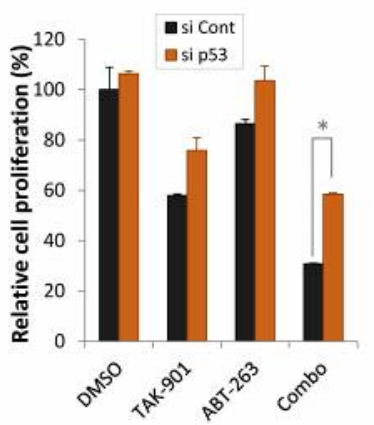

Figure 3. TAK-901 increases BCL2-associated X apoptosis regulator (BAX) in HCT116 cells in a p53-dependent manner. A: p53, BAX, BCL2 antagonist/killer 1 (BAK), p21, phospho-histone H3, and glyceraldehyde-3-phosphate dehydrogenase (GAPDH) were detected by western blotting $24 \mathrm{~h}$ after TAK-901 treatment of HCT116 cells. B, C: Expression of BAK, BAX, and p53 downstream genes was detected by TaqMan analysis 48 hours after TAK-901 treatment of HCT116 cells. D, E: HCT116 cells were reversely transfected with control or p53 siRNAs for 24 h and treated with DMSO or 200 nM TAK-901 for an additional 24 h. p53, p21, BAX, and GAPDH were detected by western blotting and expression of p53 downstream genes was detected by TaqMan analysisF: HCT116 cells were reversely transfected with control or p53 siRNAs for 24 hours and then treated with $100 \mathrm{nM}$ TAK-901 with/without $250 \mathrm{nM}$ ABT-263. After $72 \mathrm{~h}$, cell proliferation was determined (Student's t-test: ${ }^{*} p<0.01$ ).

renders HCT116 cells sensitive to apoptosis by increasing the expression of BAX through p53.

To clarify the mechanism of apoptosis induced by the combination treatment, we silenced $B A X$ or $B A K$ expression in combination with TAK-901 and ABT-263 treatment in HCT116 cells. Apoptosis induction and subsequent cell growth inhibition by the combination treatment were cancelled by BAX but not BAK depletion, indicating that BAX is indispensable for the combination effect. In HCT116 cells, we observed that BCL-xL is bound to BAX. BCL-xL is a pro-survival BCL2 family protein that binds to BAX and inhibits its pro-apoptotic activity at the mitochondrial outer membrane. Therefore, although TAK-901 induces BAX activation, BCL-xL binds to BAX and suppresses apoptosis induction. Once BAX is released from BCL-xL by ABT-263 or BCL-xL depletion, apoptosis is evoked in cancer cells. That is, BCL-xL rescues cells from cell growth inhibition induced by TAK-901 and therefore, cancer cells treated with TAK-901 are vulnerable to BCL-xL inhibition.
We observed synergism between TAK-901 and BCL-xL inhibition by both siRNAs and the inhibitors ABT-263 and gossypol in the HCT116 cell line. Synergistic cell-growth inhibition by aurora $\mathrm{B}$ and $B C L-x L$ knock-down and the selective aurora B inhibitor AZD152 was also demonstrated. This result is consistent with a previous report by Shah et al. (21).

The combination effect of TAK-901 and ABT-263 was observed in multiple cancer cell lines, but it was not synergistic in all. The synergistic effect was also observed in A549, a non-small cell lung cancer cell line that expresses wild-type $p 53$. Although total BAX expression was not increased by TAK-901 in A549 cells, active BAX and binding of BAX to BCL-xL were increased by TAK-901, suggesting that BAX is not always activated by p53 in cancer cells. The presence or absence of all possible determinants of the synergistic effect in each cell line was not elucidated; however, we demonstrated that activation of BAX is key to the combination effect. As BAX activity is 
A

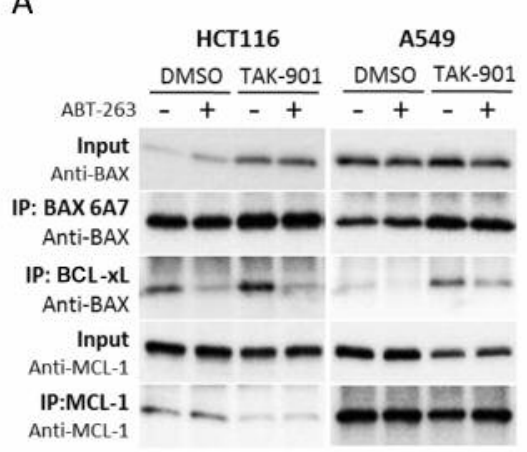

B

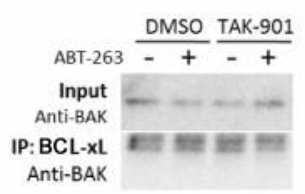

C

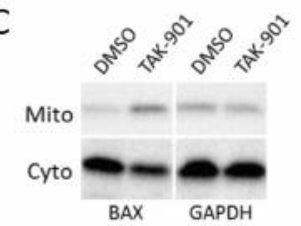

D

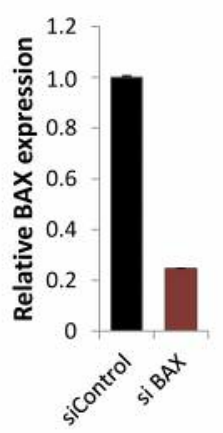

E

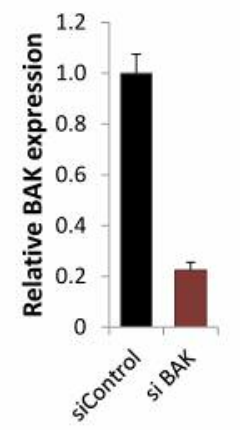

F

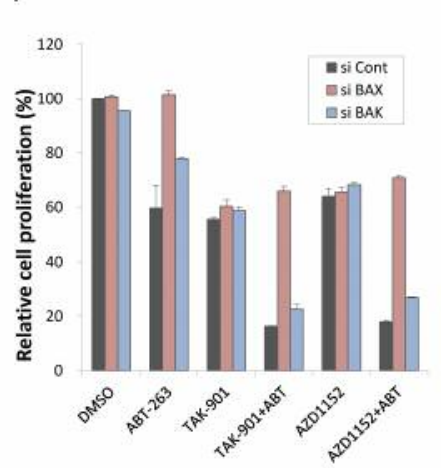

G

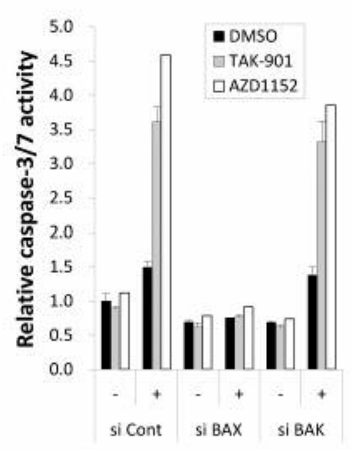

$\mathrm{H}$

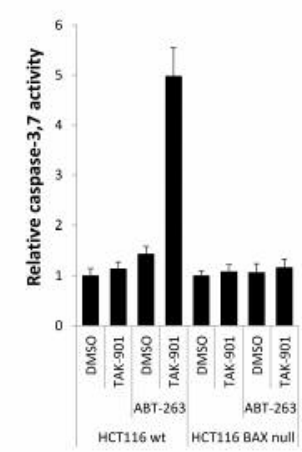

I

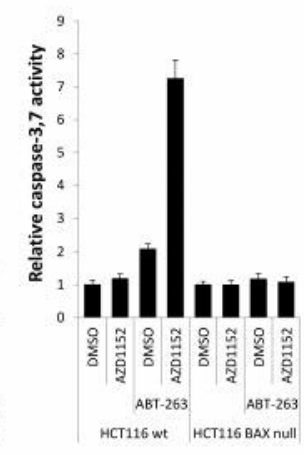

J

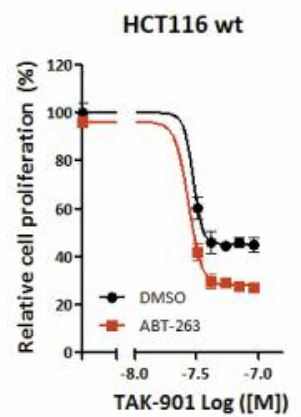

HCT116 BAX null

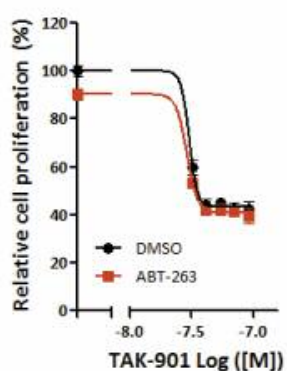

K
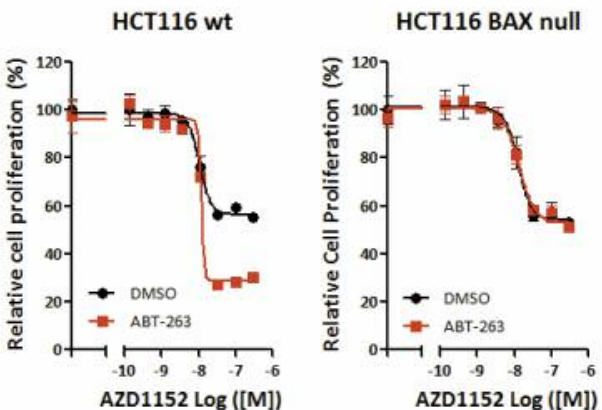

Figure 4. B-Cell lymphoma-extra large (BCL-xL) binds to BCL2-associated X apoptosis regulator (BAX) and regulates BAX-dependent apoptosis of HCT116 cells. A, B: HCT116 and A549 cells were treated with 100 nM TAK-901 with/without $250 \mathrm{nM}$ ABT-263 for 48 h and then lysed. Endogenous BAX, BCL-xL, or BCL2 family apoptosis regulator (MCL1), were immunoprecipitated from the lysates with the corresponding antibody. $B A X, B A K$, and MCL1 in lysate input and immunoprecipitated samples were detected by western blotting. $C$ : The mitochondrial fraction was separated from the cytoplasmic fraction of HCT116 cells after 24-h treatment with TAK-901. BAX and glyceraldehyde-3-phosphate dehydrogenase $(G A P D H)$ proteins were detected by western blotting. D, E: HCT116 cells were reversely transfected with control, BAX, or BCL2 antagonist/killer $1(B A K)$ siRNA. After 48-h transfection, BAX and BAK mRNA levels were measured by TaqMan analysis. F, G: After 24- $h$ transfection, the cells were treated with TAK-901 or AZD1152-HQPA with/without ABT-263 for $72 \mathrm{~h}$, after which cell proliferation and caspase-3/7 activity were measured. H, I, J, K: HCT116 wild-type BAX (HCT116 wt) and BAX-/- (HCT116 BAX null) cell lines were treated with TAK-901 or AZD1152-HQPA (100 $n M$ for caspase-3/7 assay) with/without $250 \mathrm{nM} A B T-263$. After $72 \mathrm{~h}$, cell proliferation and caspase-3/7 activity were determined.

regulated by several mechanisms at the mRNA and posttranslational levels (20), BAX may be activated by TAK-901 in different ways depending on the cellular context.

In conclusion, we identified BCL-xL inhibition as vulnerability of polyploid cells induced by TAK- 901 through a synthetic lethal siRNA screen. Our findings elucidated that induction of active BAX is a key mechanism of the revealed combination effect. Treatment with TAK-901, or other aurora B inhibitors, and BCL-xL inhibitors such as ABT-263 represents a promising biologically-relevant combination 
strategy to treat cancer in the clinic. Our study demonstrates that siRNA screening is valuable in elucidating cellular vulnerability under compound-treated conditions and in identifying targets for a biologically relevant combination strategy for anticancer therapies.

\section{Acknowledgements}

The Authors would like to thank Mark Hixon for providing the software tool to analyse inhibitor combination effects; Koji Yamamoto for integration of siRNA screen data; Koji Yoshimura, Yuichi Hikichi, Osamu Nakanishi, Hiroshi Miyake, and Christopher Claiborne for guidance and support during the course of this work.

\section{References}

1 Carmena $\mathrm{M}$ and Earnshaw WC: The cellular geography of aurora kinases. Nat Rev Mol Cell Biol 4: 842-854, 2003.

2 Ruchaud S, Carmena M and Earnshaw WC: Chromosomal passengers: conducting cell division. Nat Rev Mol Cell Biol 8: 798-812, 2007.

3 Ota T, Suto S, Katayama H, Han ZB, Suzuki F, Maeda M, Tanino $\mathrm{M}$, Terada $\mathrm{Y}$ and Tatsuka $\mathrm{M}$ : Increased mitotic phosphorylation of histone $\mathrm{H} 3$ attributable to AIM-1/aurora-B overexpression contributes to chromosome number instability. Cancer Res 62: 5168-5177, 2002.

4 Lucena-Araujo AR, de Oliveira FM, Leite-Cueva SD, dos Santos GA, Falcao RP and Rego EM: High expression of AURKA and AURKB is associated with unfavorable cytogenetic abnormalities and high white blood cell count in patients with acute myeloid leukemia. Leuk Res 35: 260-264, 2011.

5 Pohl a, Azuma M, Zhang W, Yang D, Ning Y, Winder T, Danenberg $\mathrm{K}$ and Lenz H-J: Pharmacogenetic profiling of aurora kinase $\mathrm{B}$ is associated with overall survival in metastatic colorectal cancer. Pharmacogenomics J 11: 93-99, 2011.

6 Farrell P, Shi L, Matuszkiewicz J, Balakrishna D, Hoshino T, Zhang L, Elliott S, Fabrey R, Lee B, Halkowycz P, Sang B, Ishino S, Nomura T, Teratani M, Ohta Y, Grimshaw C, Paraselli $\mathrm{B}$, Satou $\mathrm{T}$ and de Jong R: Biological characterization of TAK901, an investigational, novel, multi-targeted aurora $\mathrm{b}$ kinase inhibitor. Mol Cancer Ther 12: 460-470, 2013.

7 Wilkinson RW, Odedra R, Heaton SP, Wedge SR, Keen NJ, Crafter C, Foster JR, Brady MC, Bigley A, Brown E, Byth KF, Barrass NC, Mundt KE, Foote KM, Heron NM, Jung FH, Mortlock A a, Boyle FT and Green S: AZD1152, a selective inhibitor of aurora B kinase, inhibits human tumor xenograft growth by inducing apoptosis. Clin Cancer Res 13: 3682-3688, 2007.

8 Harrington E a, Bebbington D, Moore J, Rasmussen RK, AjoseAdeogun AO, Nakayama T, Graham J a, Demur C, Hercend T, Diu-Hercend A, Su M, Golec JMC and Miller KM: VX-680, a potent and selective small-molecule inhibitor of the aurora kinases, suppresses tumor growth in vivo. Nat Med 10: 262-267, 2004.
9 Bavetsias V and Linardopoulos S: Aurora kinase inhibitors: Current status and outlook. Front Oncol 5: 278, 2015.

10 Kaelin WG: The concept of synthetic lethality in the context of anticancer therapy. Nat Rev Cancer 5: 689-698, 2005.

11 Luo J, Emanuele MJ, Li D, Creighton CJ, Schlabach MR, Westbrook TF, Wong KK and Elledge SJ: A Genome-wide RNAi screen identifies multiple synthetic lethal interactions with the RAS oncogene. Cell 137: 835-848, 2009.

12 Whitehurst AW, Bodemann BO, Cardenas J, Ferguson D, Girard L, Peyton M, Minna JD, Michnoff C, Hao W, Roth MG, Xie X$\mathrm{J}$ and White $\mathrm{M}$ a: Synthetic lethal screen identification of chemosensitizer loci in cancer cells. Nature 446: 815-819, 2007.

13 Birsoy K, Possemato R, Lorbeer FK, Bayraktar EC, Thiru P, Yucel B, Wang T, Chen WW, Clish $\mathrm{CB}$ and Sabatini DM: Metabolic determinants of cancer cell sensitivity to glucose limitation and biguanides. Nature 508: 108-112, 2014.

14 Tse C, Shoemaker AR, Adickes J, Anderson MG, Chen J, Jin S, Johnson EF, Marsh KC, Mitten MJ, Nimmer P, Roberts L, Tahir SK, Xiao Y, Yang X, Zhang H, Fesik S, Rosenberg SH and Elmore SW: ABT-263: A potent and orally bioavailable BCL2 family inhibitor. Cancer Res 68: 3421-3428, 2008.

15 Mortlock AA, Foote KM, Heron NM, Jung FH, Pasquet G, Lohmann JJM, Warin N, Renaud F, De Savi C, Roberts NJ, Johnson T, Dousson CB, Hill GB, Perkins D, Hatter G, Wilkinson RW, Wedge SR, Heaton SP, Odedra R, Keen NJ, Crafter C, Brown E, Thompson K, Brightwell S, Khatri L, Brady MC, Kearney S, McKillop D, Rhead S, Parry T and Green S: Discovery, synthesis, and in vivo activity of a new class of pyrazoloquinazolines as selective inhibitors of aurora B kinase. J Med Chem 50: 2213-2224, 2007.

16 Honma K, Ochiya T, Nagahara S, Sano A, Yamamoto H, Hirai $\mathrm{K}$, Aso Y and Terada M: Atelocollagen-based gene transfer in cells allows high-throughput screening of gene functions. Biochem Biophys Res Commun 289: 1075-1081, 2001.

17 Hixon MS: Drug combination studies: theory and analysis. Cancer Res 70(8 Suppl): Abstract nr LB-31, 2010.

18 Yoshikawa T, Uchimura E, Kishi M, Funeriu DP, Miyake M and Miyake J: Transfection microarray of human mesenchymal stem cells and on-chip siRNA gene knockdown. J Control Release 96: 227-232, 2004.

19 Aylon Y and Oren M: P53: Guardian of ploidy. Mol Oncol 5: 315-323, 2011.

20 Luna-Vargas MPA and Chipuk JE: The deadly landscape of proapoptotic BCL2 proteins in the outer mitochondrial membrane. FEBS J: 1-14, 2016.

21 Shah OJ, Lin X, Li L, Huang X, Li J, Anderson MG, Tang H, Rodriguez LE, Warder SE, McLoughlin S, Chen J, Palma J, Glaser KB, Donawho CK, Fesik SW and Shen Y: BCL-XL represents a druggable molecular vulnerability during aurora $\mathrm{B}$ inhibitor-mediated polyploidization. Proc Natl Acad Sci USA 107: 12634-12639, 2010.

Received December 10, 2016

Revised January 20, 2017

Accepted January 24, 2017 